\title{
Impact of seconds to minutes photoperiods on Chlorella vulgaris growth rate and chlorophyll $a$ and $b$ content
}

\author{
Wendie Levasseur \& Behnam Taidi \& Robin Lacombe \& Patrick Perré \& Victor Pozzobon \\ LGPM, CentraleSupélec, Université Paris-Saclay, SFR Condorcet FR CNRS 3417, \\ Centre Européen de Biotechnologie et de Bioéconomie (CEBB), \\ 3 rue des Rouges Terres 51110 Pomacle, France
}

\begin{abstract}
In order to determine the impact of photoperiod duration, Chlorella vulgaris was studied in low cell density batch culture for seven days. Different photoperiod cycles ranging from minutes to seconds intervals were applied and their impact on microalgae cells growth and chlorophyll $a$ and $b$ contents were compared against a reference culture that was continuously illuminated. The selected photoperiods are typical of those due to alternance of dark and light zones along the algae trajectories inside photobioreactors. Throughout this study, all cultures received the same total amount of photons. When photoperiods of minutes duration were applied, the cells grew more slowly $\left(0.052 \pm 0.003 \mathrm{~h}^{-1}\right)$ but contained higher average contents of chlorophyll pigments $\left(0.27 \pm 0.03\right.$ pg.cell $\left.{ }^{-1}\right)$ than those cells grown under continuous illumination $\left(0.062 \pm 0.003 \mathrm{~h}^{-1}\right.$ and $0.42 \pm 0.08$ pg.cell ${ }^{-1}$, respectively). Cultures exposed to photoperiods of seconds duration grew the fastest $(0.072 \pm 0.002$ $\left.\mathrm{h}^{-1}\right)$ with fewer photosynthetic units $\left(0.61 \pm 0.08 \mathrm{pg}^{\left.-c^{-1} l^{-1}\right)}\right.$ than the cells grown with continuous illumination. These results suggest that light harvesting systems have a higher efficiency with photoperiods of duration in the seconds range.
\end{abstract}

Keywords: Microalgae, Light, Photoperiod, Growth rate, Chlorophyll, Photosynthesis efficiency

\section{Introduction}

Two different culture methods exist for growing microalgae: open ponds and closed photobioreactors [1,2]. The former provides a cost-effective large scale solution but does not support control over the growth conditions and is prone to contamination. In contrast, the latter is a relatively expensive method but allows a better control of operating conditions, including the choice of a single strain $[3,4]$. Closed photobioreactors are usually associated with high-value biochemicals production $[5,6,7]$.

Environmental factors such as incubation temperature, nutrients content, growth medium salinity, and light not only affect photosynthesis and cell biomass productivity, but also influence cellular metabolism and composition [8,9]. The first three parameters can be optimized relatively easily while lighting conditions optimisation is more challenging.

From a metabolic perspective, light is the energy source for microalgae photosynthesis and $\mathrm{CO}_{2}$ in its various forms is the carbon source. Photosynthesis comprises two steps: lights reactions occurring on the nanosecond scale transforming light energy into chemical energy; and dark reactions, also known as carbon-fixation reactions, happening on the millisecond scale and leading to $\mathrm{CO}_{2}$ fixation and production of organic molecules such as carbohydrates and lipids [10]. The latter is powered by the energy produced during the light reactions. The absorption of light and the conversion of photon energy into chemical energy takes place through photosystems II and I in the thylakoid membrane. Nevertheless, exposure of photosynthetic organisms to high light intensity may become a stress factor as it can damage photosystem II reaction centers due to limitation of electrons transfer process, causing photoinhibition and the disruption of photosynthesis [11, 12, 13, 14]. Therefore, the lighting conditions to which cultures are submitted are considered to be an important factor in productivity.

At full industrial scale, light limitation is one of the main problems in achieving the whole commercial potential of microalgae $[15,16]$. Regardless of the culture method, biomass production is quickly limited in moderately dense photoautotrophic cultures due to cellular self-shading $[17,18]$. This self-shading combined with the need to supply the cells with $\mathrm{CO}_{2}$ necessarily imposes photoperiods to the microalgae.

The incident light sent onto the microalgal culture surface is absorbed along the light path through the culture, resulting in a light gradient in the photobioreactor. Adequate mixing can keep the cells in suspension and allow nutrient distribution. Furthermore, it should shuttle the algal cells between the light saturating zone near the illuminated surfaces of the photobioreactor and the dark core of the culture. Thus, the algal cells experience periodic light-dark cycles. Generally, these mixing-induced cycles are about several seconds to minutes long [19], and will depend on the geometry of the photobioreactor.

Several studies have been conduced on the impact of light/dark cycles variation on microalgal response. In a pioneering approach [20], enhancement factors of photosynthesis were determined as a function of light pulses and dark periods duration. The results pointed out that intermittent light, i.e. short light flashes separated by dark periods, would allow to increase the efficiency of light utilization. More recent studies led to the conclusion that these short periodic light/dark cycles are beneficial 
Table 1: Composition of the B3N medium

\begin{tabular}{llr}
\hline & Composition & Concentration $(\mathrm{mg} / \mathrm{L})$ \\
\hline Solution A & $\mathrm{NaNO}_{3}$ & 750 \\
& $\mathrm{CaCl}_{2}, 2 \mathrm{H}_{2} \mathrm{O}$ & 25 \\
& $\mathrm{MgSO}_{4}, 7 \mathrm{H}_{2} \mathrm{O}$ & 75 \\
& FeEDTA & 20 \\
\hline Solution B & $\mathrm{K}_{2} \mathrm{HPO}_{4}$ & 75 \\
& $\mathrm{KH}_{2} \mathrm{PO}_{4}$ & 175 \\
& $\mathrm{NaCl}$ & 20 \\
\hline Solution C & $\mathrm{H}_{3} \mathrm{BO}_{3}$ & 2.86 \\
& $\mathrm{MnCl}_{2}, 4 \mathrm{H}_{2} \mathrm{O}$ & 1.81 \\
& $\mathrm{ZnSO}_{4}, 7 \mathrm{H}_{2} \mathrm{O}$ & 0.22 \\
& $\mathrm{CuSO}_{4}, 7 \mathrm{H}_{2} \mathrm{O}$ & 0.08 \\
& $\mathrm{MoO}_{3} 85 \%$ & 0.036 \\
& $\mathrm{CoSO}_{4}, 7 \mathrm{H}_{2} \mathrm{O}$ & 0.09 \\
\hline
\end{tabular}

to algal growth $[21,22,23]$. It is, however, necessary to distinguish between the fluctuating light regime and the flashing light regime. In the first case, frequencies generally range in the order of an hour to $10 \mathrm{~Hz}$ [24] whereas flashing light regimes refer to higher frequencies (above $100 \mathrm{~Hz}$ ).

The aim of the study is to determine the effect of different photoperiod durations on the microalgae growth under emulated photobioreactor conditions. To do so, photoautotrophic cultures of $C$. vulgaris were grown at different photoperiods over 7 day-long experiments prior to significant auto-shadowing. Throughout the entire experiments, the cultures were never exposed to complete darkness to prevent metabolism of internal carbon and energy reserves from taking place [25]. Therefore, the light intensity in the experiments ranged from $350 \mu \mathrm{mol}$ quanta. $\mathrm{m}^{-2} \cdot \mathrm{s}^{-1}$ to only $80 \mu \mathrm{mol}$ quanta.m ${ }^{-2} \cdot \mathrm{s}^{-1}$. The applied photoperiod cycles varied from the minute to the second range. The cell concentration was measured twice daily and the chlorophyll $a$ and $b$ contents were determined at the end of each experiment.

\section{Materials and Methods}

\subsection{Strain and culture medium}

C. vulgaris (SAG 12A) was obtained from the culture collection at Göttingen University (SAG).

The cells were grown in modified B3N medium which composition is detailed in Table 1. The modified B3N medium is made from three solutions providing different minerals elements to prevent nutrient limitation [26]. These solutions were prepared beforehand and stored at $4^{\circ} \mathrm{C}$ in the dark.

\subsection{Culture procedure}

The C. vulgaris inoculum (preculture) was grown in modified B3N medium for 7 days in shake-flask culture at $25^{\circ} \mathrm{C}$ before being used to inoculate the experimental cultures. An orbiting platform at $150 \mathrm{rpm}$ was used for the preparation of the inoculum and the experiments themselves.

The experimental cultures (50 mL B3N medium in $250 \mathrm{~mL}$ total volume) were inoculated using the preculture and subsequently incubated as described above for seven days. The initial cell densities were set at $5 \times 10^{5}$ cells $/ \mathrm{mL}$.

For each photoperiod, six replicates of microalgae cultures were performed. A seventh flask containing blank culture medium was added as control. Experiments lasted for 7 days to avoid culture overgrowth that would induce mutual shading $[27]$.

\subsection{Experimental setup}

C. vulgaris was grown as described above in shake-flask photobioreactors.

The lighting differed from that used for the preparation of the inocula (147 $\mu$ mol quanta.m $\left.{ }^{-2} \cdot \mathrm{s}^{-1}\right)$. The experimental cultures were alternatively illuminated with light provided by a LEDs panel positioned above them $(40 \mathrm{~cm})$. The flasks were incubated in a photoperiod chamber at $20^{\circ} \mathrm{C}$ (Figure 1) preventing penetration of external light and ensuring that LEDs act as the sole light source. 


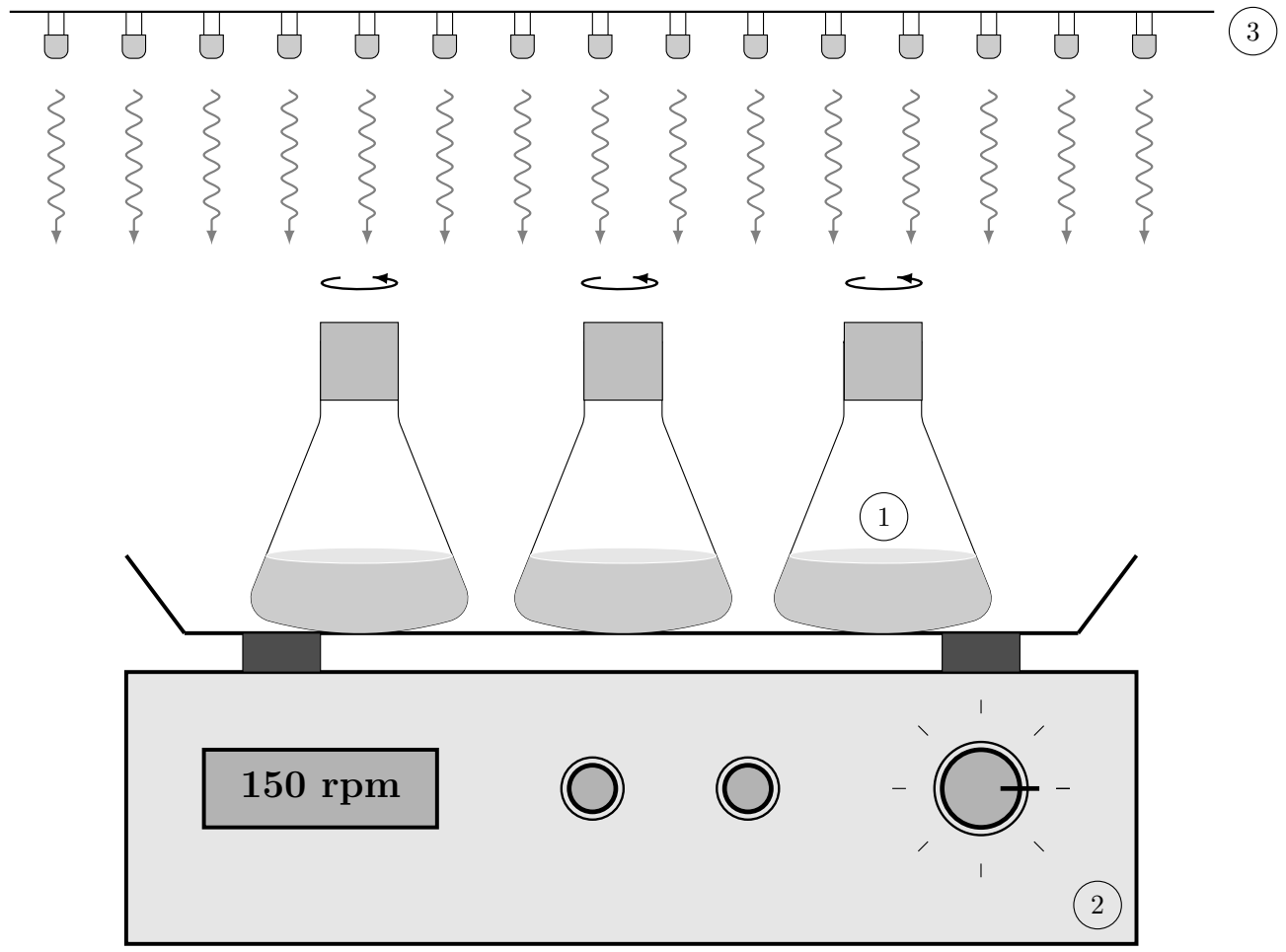

Figure 1: Experimental set-up. (1) Microalgae culture flasks placed on a tray, (2) Orbital shaker agitating the flasks, (3) Lighting LED panel.

\subsection{Illumination and photoperiod protocol}

In a preliminary phase of this work, $C$. vulgaris cultures were exposed to continuous illumination of various intensities. A light intensity of $215 \mu \mathrm{mol}$ quanta.m $\mathrm{m}^{-2} \cdot \mathrm{s}^{-1}$ at the surface of the cultures was determined to be high enough to promote growth and low enough to avoid excessive photodamage. The results under a continuous lighting of $215 \mu$ mol quanta.m ${ }^{-2} . \mathrm{s}^{-1}$ were used as a reference for the following tests.

Then, the microalgal cells were exposed to intermittent light cycles of equal light and dark intervals (Figure 2). The bright period corresponds to a maximal intensity of $350 \mu \mathrm{mol}$ quanta. $\mathrm{m}^{-2} \cdot \mathrm{s}^{-1}$ and the dark periods to about one five of the brightness of the maximal intensity, i.e. an intensity of $80 \mu \mathrm{mol}$ quanta. $\mathrm{m}^{-2} \cdot \mathrm{s}^{-1}$. The different light cycles were conduced with an equal average total light intensity, similar to the reference test (Table 2).

Table 2: Experimental conditions

\begin{tabular}{|c|c|c|c|}
\hline Cycle duration (s) & $\begin{array}{l}\text { Light phase duration }(\mathrm{s}) \\
\times \text { Light intensity }(\mu \mathrm{mol} \\
\left.\text { quanta. } \mathrm{m}^{-2} \cdot \mathrm{s}^{-1}\right)\end{array}$ & $\begin{array}{l}\text { Dark phase duration }(\mathrm{s}) \\
\times \text { Light intensity }(\mu \mathrm{mol} \\
\left.\quad \text { quanta. } \mathrm{m}^{-2} . \mathrm{s}^{-1}\right)\end{array}$ & $\begin{array}{l}\text { Average light intensity } \\
\text { over a cycle }(\mu \mathrm{mol} \\
\left.\text { quanta.m }{ }^{-2} \cdot \mathrm{s}^{-1}\right)\end{array}$ \\
\hline 1 & $0.5 \times 350$ & $0.5 \times 80$ & 215 \\
\hline 2 & $1 \times 350$ & $1 \times 80$ & 215 \\
\hline 4 & $2 \times 350$ & $2 \times 80$ & 215 \\
\hline 120 & $60 \times 350$ & $60 \times 80$ & 215 \\
\hline 240 & $120 \times 350$ & $120 \times 80$ & 215 \\
\hline Continuous & - & - & 215 \\
\hline
\end{tabular}

C. vulgaris was exposed to seven different illumination regimes: 0.5 second, 1 second, 2 seconds, 60 seconds, and 120 seconds. The total light received by all cultures during the seven days of incubation was always the same.

In a reference experiment, C. vulgaris cultures were exposed to continuous illumination at $215 \mu$ mol at the surface of the cultures in an attempt to prevent photodamage during the early stages of cultures development. 

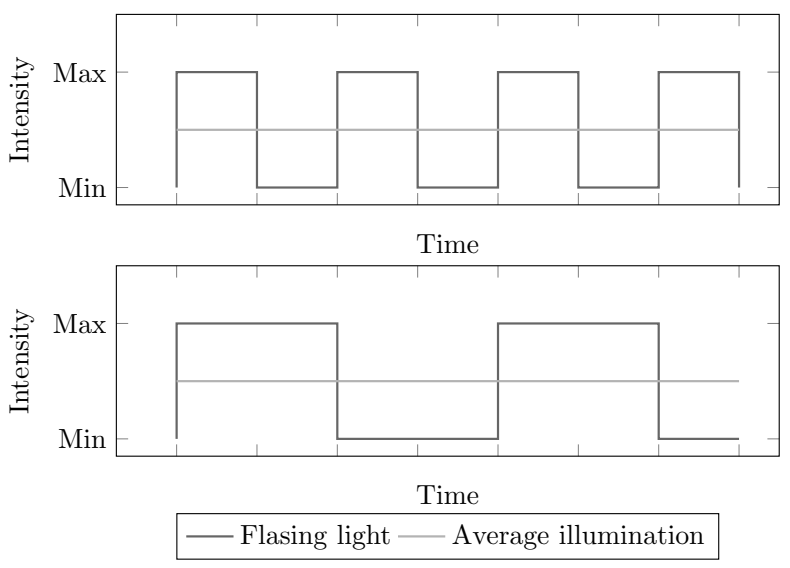

Figure 2: Schematic diagram of continuous light and different periods of fluctuating light with an equal average intensity

\subsection{Cell growth measurements}

Cell growth was followed on a continual basis ( $1 \mathrm{~mL}$ sample collected twice per day) by following the absorbance of the culture at $800 \mathrm{~nm}$ and the enumeration of the cell concentration (Beckman Coulter Multisizer 4). The samples were always diluted to the same concentration $\left(\mathrm{A}_{800}\right.$ of $\left.0.2-0.4\right)$ before the cell count so that the same concentration of cells was always presented to the Coulter counter. Cell counts were performed in triplicate on the same sample and the standard deviation was used as an indication of the error in the enumeration method.

After seven days of incubation, the cultures were harvested and dry weights biomass concentration of the cultures were determined by the following method. The wet biomass was separated from the supernatant by centrifugation at $6738 \mathrm{~g}$ for 10 minutes at $2^{\circ} \mathrm{C}$. The pellet was washed with the same volume of distilled water. In order to wash the pellet, it was resuspended and subjected to centrifugation. This process was repeated twice and the pellet was dried at $105^{\circ} \mathrm{C}$ overnight.

\subsection{Chlorophyll content determination}

Chlorophyll $a$ and $b$ were quantified using a spectrophotometric method $[28,29,30,31]$.

Approximately $15 \mathrm{~mL}$ of fresh algal culture was centrifuged $\left(10\right.$ minutes, $\left.6738 \mathrm{~g}, 2^{\circ} \mathrm{C}\right)$. The pellet was then frozen at $-18^{\circ} \mathrm{C}$ overnight and lyophilized. About $1 \mathrm{mg}$ of powder was weight precisely, mixed with water and centrifuged (10 minutes, 15294 $\mathrm{g}, 8^{\circ} \mathrm{C}$ ). The pellet was then mixed with a solution composed of $85 \%$ methanol and $15 \%$ water with 1.5 mmol/L of sodium dithionite. The mixture was placed in a water bath protected from light and heated at $40^{\circ} \mathrm{C}$ for 32 minutes. Finally, the pellet was separated by centrifugation $\left(10\right.$ minutes, $\left.15294 \mathrm{~g}, 8^{\circ} \mathrm{C}\right)$ in order to remove the cell debris whilst keeping the total chlorophyll in the supernatant. The supernatant absorbance was determined at 650 and 664 nm wavelengths corresponding to absorption maxima of chlorophyll $\mathrm{b}$ and chlorophyll a, respectively. The absorbance measurements were performed against $85 \%$ methanol as a blank. Chlorophyll $a$ and $b$ concentrations were calculated in mg/L (Eq. 1 and 2) [32]:

$$
\begin{aligned}
\text { Chl a } & =16.41 A_{664}-8.09 A_{650} \\
\text { Chl b } & =30.82 A_{650}-12.57 A_{664}
\end{aligned}
$$

\section{Results}

In order to evaluate the impact of photoperiod duration on $C$. vulgaris growth rate and photosynthetic pigments content, the experiments were performed in six replicates. The results are expressed as the average obtained from the six parallel cultures with their respective standard deviations.

At the end of each culture, the final cell concentration reached $2.2 \times 10^{7}$ cells $/ \mathrm{mL}$. Over the course of all the experiments, the average cell size remained constant at about $3.8 \mu \mathrm{m}$.

\subsection{Biomass production}

Dry weight values of $C$. vulgaris after seven days of culture under different light treatments are presented in Figure 3 . Under continuous illumination, $C$. vulgaris reached $0.52 \pm 0.01 \mathrm{~g} / \mathrm{L}$ of dry biomass. The dry weight increased with the light frequency reaching the highest value of $0.619 \pm 0.002 \mathrm{~g} / \mathrm{L}$ for a 0.5 -second photoperiod.

In all experiements, the dry weight content was below $1 \mathrm{~g} / \mathrm{L}$, which means that the cultures were optically non-dense. The 


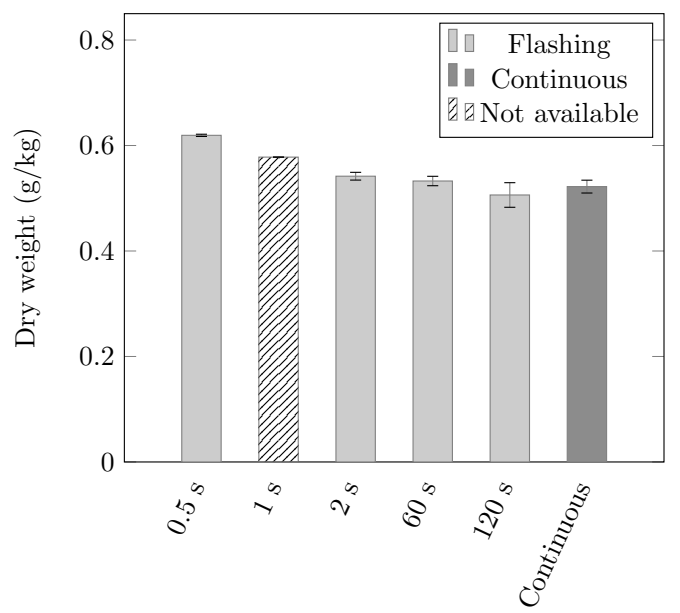

Figure 3: Dry weight under different photoperiods after 7 days of culture; data shown as mean \pm SD $(\mathrm{n}=6)$

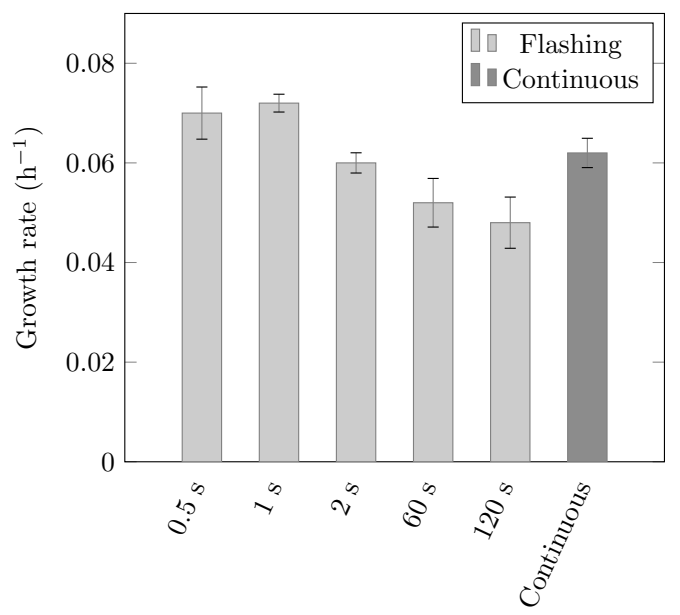

Figure 4: Growth rate under different photoperiods after 7 days of culture; data shown as mean \pm SD $(n=6)$

experiments were carried out in order to maintain low cell density in the culture throughout the experiment duration to insure an homogeneous light field. Thus, as mutual shading was negligible, each cell was exposed to the same amount of light.

\subsection{Growth rate}

Figure 4 displays the growth rates of $C$. vulgaris under the different light conditions.

Under continuous illumination, the value of the specific growth rate $(\mu)$ was $0.062 \pm 0.003 \mathrm{~h}^{-1}$.

Regarding the photoperiod of minutes duration, i.e. under light periods of 60 and 120 seconds, specific growth rates presented values lower than that found under continuous illumination. The lowest value was reached for a light exposure time of 120 seconds $\left(0.048 \pm 0.005 \mathrm{~h}^{-1}\right)$.

For the photoperiod of the order of a second, C. vulgaris showed specific growth rates higher than that observed under continuous illumination. The highest value reached was $0.072 \pm 0.002 \mathrm{~h}^{-1}$ for a light exposure time of 0.5 second.

\subsection{Chlorophyll content and photosynthesis enhancement}

Chlorophyll a and b contents under different lighting conditions are depicted in Figure 5.

The chlorophyll contents per cell are given in Figure 5a. Under continuous illumination, it was $0.42 \pm 0.08$ pg.cell ${ }^{-1}$. Regarding the fluctuating light conditions, for photoperiods of the order of a minute, the chlorophyll content was higher than the one under continuous illumination with the highest content of $0.61 \pm 0.08$ pg.cell ${ }^{-1}$ reached for the light exposure time of 60 seconds. As for photoperiods of the order of a second, the chlorophyll content was lower than that observed under 


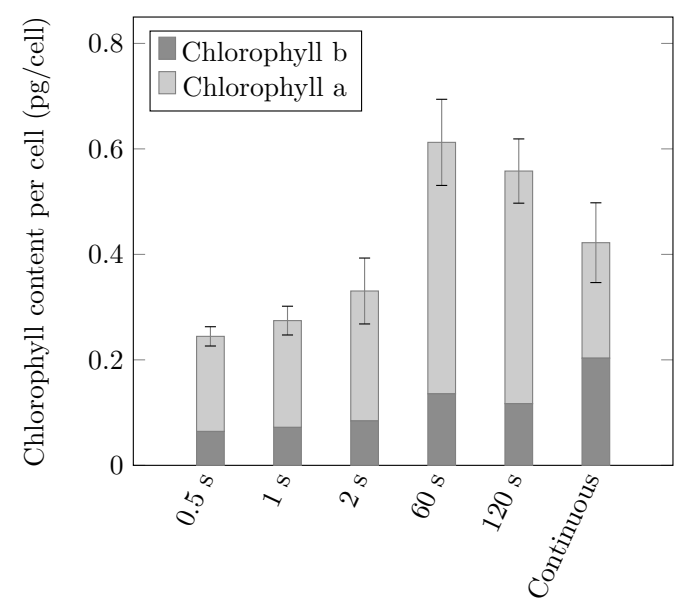

(a) General trend of the chlorophyll content

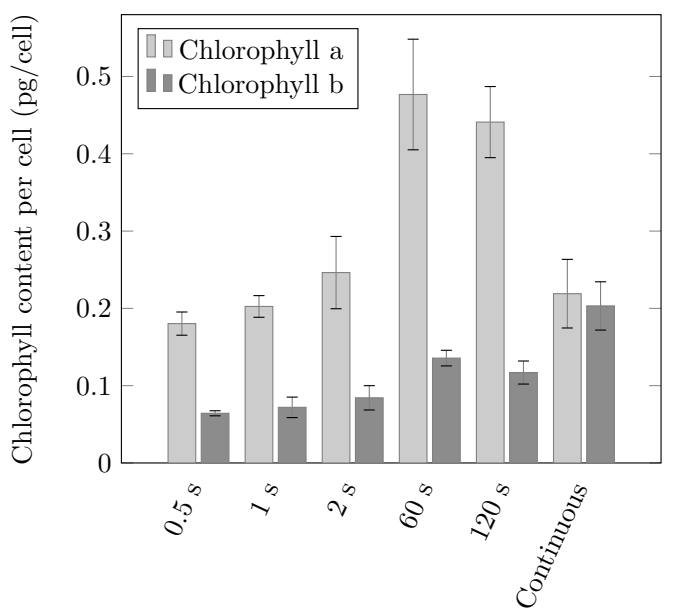

(b) Separate display of chlorophyll a and b contents

Figure 5: Chlorophyll a and b content under different photoperiods after 7 days of culture; data shown as mean \pm SD $(n=6)$

continuous light. The lowest value was obtained for a light exposure time of 0.5 second $\left(0.24 \pm 0.02\right.$ pg.cell $\left.{ }^{-1}\right)$.

Focusing on the chlorophyll $a$ and the chlorophyll $b$ individual contents, different patterns are observed (Figure 5b). Under continuous illumination, the chlorophyll $a$ and chlorophyll $b$ contents were nearly at the same level. At the end of the experiment, C. vulgaris cells contained $0.22 \pm 0.04$ pg.cell ${ }^{-1}$ and $0.20 \pm 0.03$ pg.cell ${ }^{-1}$ of chlorophyll $a$ and chlorophyll $b$, respectively. Regarding photoperiods of the order of a minute, the chlorophyll $a$ content was higher than the one obtained under continuous illumination, reaching the maximum value of $0.48 \pm 0.07$ pg.cell ${ }^{-1}$ for the light photoperiod of 60 seconds. Conversely, for the same light exposure conditions, the chlorophyll $b$ content is lower than the one obtained under continuous illumination. Cell reached the lowest value of $0.12 \pm 0.02$ pg.cell ${ }^{-1}$ for the light photoperiod of 120 seconds. As for photoperiods below 60 seconds, the chlorophyll $a$ content is relatively the same as the one observed under continuous light $\left(0.22 \pm 0.04\right.$ pg.cell $\left.{ }^{-1}\right)$. As regards the chlorophyll $b$ content, for the same light exposure conditions, the same trend as for photoperiods above 60 seconds is observed. Indeed, the chlorophyll $b$ content is lower than that under continuous illumination, reaching the lowest value of $0.064 \pm 0.003$ pg.cell ${ }^{-1}$ for the light photoperiod of 0.5 second.

Developing the analysis of the above results further, a photosynthetic yield was calculated by dividing the growth rate by the number of photosynthetic units contained in each cell (Figure 6). This yield could give information about the photosynthesis efficiency by dividing the biomass production by the harvested energy.

Under continuous illumination, the photosynthesis yield was $0.15 \mathrm{~h}^{-1} /\left(\mathrm{pg}\right.$.cell $\left.{ }^{-1}\right)$. At photoperiods of 60 seconds and above, this yield was 0.6 times lower that the one under continuous illumination. The lowest value reached $0.09 \mathrm{~h}^{-1} /\left(\mathrm{pg} . \mathrm{cell}{ }^{-1}\right)$ was observed with photoperiods of 60 and 120 seconds. It means that photosynthesis is less efficient at these photoperiods since a lower amount of biomass is produced per photosynthetic units. For short photoperiods, the photosynthetic yield is 1.7 to 1.9 times higher than the one obtained under continuous illumination. Furthermore, it further increases with short time of light exposures, reaching the highest value of $0.29 \mathrm{~h}^{-1} /\left(\mathrm{pg}\right.$.cell $\left.{ }^{-1}\right)$ for a 0.5 second-photoperiod. These results indicate that photosynthesis is more efficient that under continuous illumination.

\subsection{Confirming photodamages}

The above results suggest photodamage as a valid explanation for the observed trends in terms of both growth rate and chlorophyll contents. In order to assess for their induction, an experiment at maximal light intensity of $350 \mu \mathrm{mol} \mathrm{quanta.m}^{-2} . \mathrm{s}^{-1}$ was performed. The results indicate a $17 \%$ lower growth rate when exposed to the highest light intensity. The chlorophyll $a$ content is also affected by the increased light intensity. Under maximal light intensity, it is $16 \%$ lower than the one under 215 $\mu$ mol quanta. $\mathrm{m}^{-2} \cdot \mathrm{s}^{-1}$. In addition, no significant changes were observed for the chlorophyll $b$ content, leading to an increased chlorophyll ratio ( $b$ over $a$ ) under the highest illumination. It indicates an increased relative content of photoprotective pigments when exposed to $350 \mu \mathrm{mol}$ quanta. $\mathrm{m}^{-2} \cdot \mathrm{s}^{-1}$. These results highlight the presence of photodamage induced by the high intensity lighting used in this work.

\section{Discussion}




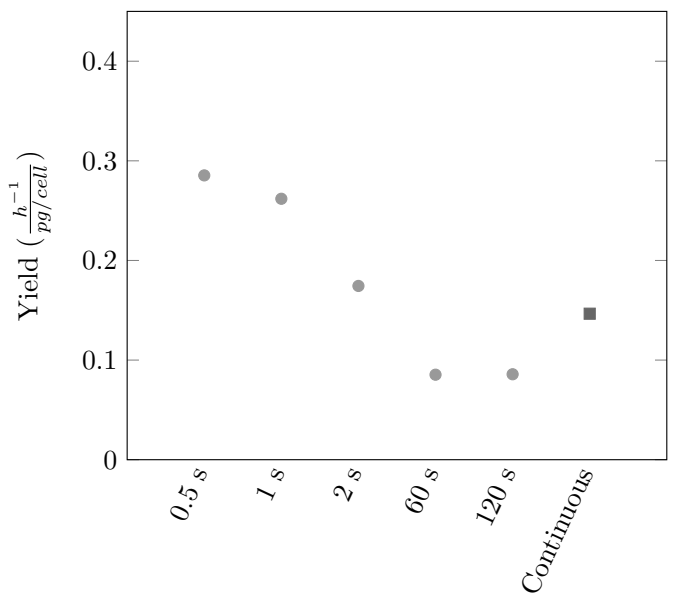

Figure 6: Photosynthetic efficiency under different photoperiods after 7 days of culture

From these results, two distinct patterns emerge (Fig. 5a and 5b). The first one, from 0.5 to 2 seconds cycles, exhibits higher culture growth rate and lower cell chlorophyll $b$ content than continuous illumination. The second one, from 1 and 2 minutes cycles, shows lower culture growth rate, significantly higher cell chlorophyll $a$ content and lower chlorophyll $b$ content than continuous illumination.

Keeping in mind that from a metabolic perspective, cells must maintain a balance between the energy derived from the light reactions in the chloroplast and the amount of energy used during carbon fixation and other processes. Changes in environmental conditions, and in particular in light conditions, upset this balance requiring microalgae to adapt.

The first pattern could be explained by a photoprotection process. Even though, intense light can damage the photosynthetic apparatus, short exposure durations are too brief to severely harm it. Indeed, when exposure to high light intensity is short, i.e. the exposure time does not exceed a few seconds, the excess of captured light energy is sent to the xanthophyll cycle and dissipated through heat [33]. This cycle is used to eliminate short terms light energy surplus which interfere with the electron flow between the two photosystems. An overexposure to light leads to the emergence of a proton gradient across the thylakoid membrane which acidifies the thylakoid lumen. Under these conditions, violaxanthin normally found in the photosynthetic membranes is converted into antheraxanthin and zeaxanthin, which are unable to transfer their energy directly to the photosystem. Hence, this trapped energy is then dissipated as heat release as part of Non-Photochemical Quenching (NPQ) mechanism. As these photoprotective pigments are sufficiently effective to manage the excess of light for short time of exposure to light, the cell does not need to produce high amount of photoprotecting pigment explaining the low chlorophyll $b$ content.

Hence the first set of conditions are favorable to culture growth in two ways:

- they decrease the amount of energy that has to be diverted to photosynthetic units reparation, redirecting this energy toward cell growth,

- they lower the need to produce chlorophyll $b$ which acts as a photoprotecting pigment. By reducing the production of chlorophyll $b$, these conditions free even more energy and metabolites for cell growth.

Such mechanisms explain why for 0.5 to 2 seconds cycles, chlorophyll $b$ content remains lower than under continuous illumination while the growth rate is higher. These results are in agreement with the litterature. Several studies reported that fast alternations between light and dark phase from less than $40 \mu$ seconds to 1 second greatly enhance photosynthetic efficiency $[34,20]$. Moreover, the findings of Grobbelaar et al. indicate that light/dark cycles with exposure times lower than 1 second contribute to an enhancement of photosynthetic rates and light utilisation efficiencies [35].

The second pattern can be seen as much more unfavorable in terms of algal growth. When extending light/dark cycles duration, that is cycles longer than 1 minute, cells could be subject to more damage as xanthophyll cycle is saturated and cannot handle the excess of light on its own. Yet another adaptation mechanism must be established. Furthermore, the light stress imposed in our study case does not seem to be long enough to trigger a proper adaptation. Indeed, cell pigment adaption can be observed throughout a day, with an hour scale response time [36]. In our case, under continuous illumination, cells have enough time to adapt to - and protect themselves from - lighting conditions by producing protective chlorophyll $b$. It is not the case for cells growing under minutes long cycles. Such conditions affect negatively growth in two ways:

- An increase in the amount of energy that has to be diverted to photosynthetic units repair, reducing the amount of energy available for cell growth,

- An increase in cells energy harvesting by increasing the number of photosynthetic units as indicated by the higher chlorophyll $a$ content. This process could divert metabolites and energy from cell growth. 
These results are consistent with those found in the litterature. Keren et al. [?] showed the presence of a plateau for the damage rate. This latter is reached for an interval between the flashes of about a minute. Moreover, Graham et al. [24] found that fluctuating light regimes ranging from 10 seconds to 100 seconds have non-beneficial effects leading to severely hampered growth. Indeed, long-term photoacclimation (hours) occures when short-term responses are insufficient for coping with the changes in light intensity. Acclimatation (production of protective chlorophyll $b$, in our case) requires extensive changes in enzymatic activity and gene expression that lead to alterations in the concentration of photosynthetic complexes and thus, changes in antenna composition [37].

Such mechanisms could explain why for 1 to 2 minutes cycles, chlorophyll $a$ content is higher than under continuous illumination, while chlorophyll $b$ content is not high enough to protect the photosynthetic unit (higher than 0.5 to 2 seconds case, lower than continuous light reference). Yet this adaptation strategy is far from optimal as it does not allow for the culture to achieve growth equivalent to the one under continuous illumination. These light/dark cycles of minute duration represent a lighting regime where the cells are stuck into a zone between photoprotection (seconds) and photoacclimation (hours).

Finally, this reasoning could be further substantiate by photosystem I and II, and xanthophyll cycle pigments concentration analyses.

\section{Conclusion}

The aim of this study was to highlight the impact of the light/dark cycle duration on microalgal growth and chlorophyll a and b contents. To do so, C. vulgaris cells were exposed to different intermittent light cycles of equal light and dark intervals ranging from 0.5 second to 120 seconds. Cultures under continuous illumination were used as a reference. Regardless of the light cycle applied, the same amount of photons lit the cultures.

The results demonstrate that the different fluctuating light periods have a significant impact on the key growth parameters monitored. Under photoperiods above one minute, cells show lower growth rates and higher photosynthetic pigments production than those performed under continuous illumination. Compared with cells grown under continuous illumination, under photoperiods of shorter than 60 seconds, cultures exhibit higher growth kinetics with lower photosynthetic pigments.

This behaviour suggests that light harvesting systems have a much higher efficiency under second scale photoperiod. From an engineering perspective, these findings could help scaling up photobioreactors. Increasing the size of a photobioreactor modifies its hydrodynamics, usually increasing the period in which cells alternate between light/dark cycles. The design of a photobiorector should take into account that these cycles should not exceed the order of seconds as to not hinder cell growth.

\section{Acknowledgements}

The authors would like to thank Département de la Marne, Région Grand Est and Grand Reims for their financial support. They also thank Rayen Filali for his technical support.

\section{Declaration of author contributions}

PP and VP initiated and planned the project. BT provided practical advice on the experimental set up and conditions to test. RL and WL performed the experiments and led the data analyses. All the authors contributed to the interpretation of the data. WL drafted the manuscript. PP, BT and VP performed critical revision of the manuscript. All the authors read and approved the final manuscript and take responsibility for the integrity of the work as a whole, from inception to finished article.

\section{Conflicts of interest}

Authors have no conflict of interest to disclose.

\section{Statement of Informed Consent, Human/Animal Rights}

No conflicts, informed consent, human or animal rights applicable. 


\section{References}

[1] O. Pulz, Photobioreactors: production systems for phototrophic microorganisms, Applied Microbiology and Biotechnology 57 (3) (2001) 287-293. doi:10.1007/s002530100702.

URL https://link.springer.com/article/10.1007/s002530100702

[2] O. Jorquera, A. Kiperstok, E. A. Sales, M. Embiruçu, M. L. Ghirardi, Comparative energy life-cycle analyses of microalgal biomass production in open ponds and photobioreactors, Bioresource Technology 101 (4) (2010) $1406-1413$. doi:10.1016/j.biortech.2009.09.038.

URL http://www.sciencedirect.com/science/article/pii/S0960852409012449

[3] G. Torzillo, B. Pushparaj, F. Bocci, W. Balloni, R. Materassi, G. Florenzano, Production of Spirulina biomass in closed photobioreactors, Biomass 11 (1) (1986) 61-74. doi:10.1016/0144-4565(86)90021-1.

URL http://www.sciencedirect.com/science/article/pii/0144456586900211

[4] I. Rawat, R. Ranjith Kumar, T. Mutanda, F. Bux, Dual role of microalgae: Phycoremediation of domestic wastewater and biomass production for sustainable biofuels production, Applied Energy 88 (10) (2011) 3411-3424. doi:10.1016/j.apenergy.2010.11.025.

URL http://www.sciencedirect.com/science/article/pii/S0306261910004885

[5] M. Stark, I. O'Gara, An Introduction to Photosynthetic Microalgae, Disruptive Science and Technology 1 (2) (2012) 65-67. doi:10.1089/dst.2012.0017.

URL http://online.liebertpub.com/doi/full/10.1089/dst.2012.0017

[6] M. R. Tredici, R. Materassi, From open ponds to vertical alveolar panels: the Italian experience in the development of reactors for the mass cultivation of phototrophic microorganisms, Journal of Applied Phycology 4 (3) (1992) $221-231$. doi:10.1007/BF02161208.

URL https://link.springer.com/article/10.1007/BF02161208

[7] G. Huang, F. Chen, D. Wei, X. Zhang, G. Chen, Biodiesel production by microalgal biotechnology, Applied Energy 87 (1) (2010) 38-46. doi:10.1016/j.apenergy.2009.06.016.

URL http://www.sciencedirect.com/science/article/pii/S0306261909002530

[8] A. Richmond, Handbook of Microalgal Culture: Biotechnology and Applied Phycology, John Wiley \& Sons, 2008.

[9] K. K. Vasumathi, M. Premalatha, P. Subramanian, Parameters influencing the design of photobioreactor for the growth of microalgae, Renewable and Sustainable Energy Reviews 16 (7) (2012) 5443-5450. doi:10.1016/j.rser.2012.06.013.

URL http://www.sciencedirect.com/science/article/pii/S1364032112003930

[10] A. A. Lunka, D. J. Bayless, Effects of flashing light-emitting diodes on algal biomass productivity, Journal of Applied Phycology 25 (6) (2013) 1679-1685. doi:10.1007/s10811-013-0044-1.

URL https://link.springer.com/article/10.1007/s10811-013-0044-1

[11] M. J. Barbosa, M. Janssen, N. Ham, J. Tramper, R. H. Wijffels, Microalgae cultivation in air-lift reactors: Modeling biomass yield and growth rate as a function of mixing frequency, Biotechnology and Bioengineering 82 (2) (2003) 170179. doi:10.1002/bit.10563.

URL http://onlinelibrary.wiley.com/doi/10.1002/bit.10563/abstract

[12] Z. Perrine, S. Negi, R. T. Sayre, Optimization of photosynthetic light energy utilization by microalgae, Algal Research 1 (2) (2012) 134-142. doi:10.1016/j.algal.2012.07.002.

URL http://www.sciencedirect.com/science/article/pii/S2211926412000288

[13] J. A. Raven, The cost of photoinhibition, Physiologia Plantarum 142 (1) (2011) 87-104. doi:10.1111/j.13993054.2011.01465.x.

[14] Z. A. Khoeyi, J. Seyfabadi, Z. Ramezanpour, Effect of light intensity and photoperiod on biomass and fatty acid composition of the microalgae, Chlorella vulgaris, Aquaculture International 20 (1) (2012) 41-49. doi:10.1007/s10499-011-9440-1. URL https://link. springer.com/article/10.1007/s10499-011-9440-1

[15] E. Jacob-Lopes, C. H. G. Scoparo, L. M. C. F. Lacerda, T. T. Franco, Effect of light cycles (night/day) on CO2 fixation and biomass production by microalgae in photobioreactors, Chemical Engineering and Processing: Process Intensification 48 (1) (2009) 306-310. doi:10.1016/j.cep.2008.04.007.

URL http://www.sciencedirect.com/science/article/pii/S0255270108001037

[16] S. Wahidin, A. Idris, S. R. M. Shaleh, The influence of light intensity and photoperiod on the growth and lipid content of microalgae Nannochloropsis sp., Bioresource Technology 129 (Supplement C) (2013) 7-11. doi:10.1016/j.biortech.2012.11.032. URL http://www.sciencedirect.com/science/article/pii/S0960852412017063

[17] M. Kula, H. M. Kalaji, A. Skoczowski, Culture density influence on the photosynthetic efficiency of microalgae growing under different spectral compositions of light, Journal of Photochemistry and Photobiology B: Biology 167 (Supplement C) (2017) 290-298. doi:10.1016/j.jphotobiol.2017.01.013.

URL http://www.sciencedirect.com/science/article/pii/S1011134416310132 
[18] J. U. Grobbelaar, Microalgal biomass production: challenges and realities, Photosynthesis Research 106 (1-2) (2010) 135-144. doi:10.1007/s11120-010-9573-5.

[19] J. U. Grobbelaar, Do light/dark cycles of medium frequency enhance phytoplankton productivity?, Journal of Applied Phycology 1 (4) (1989) 333-340. doi:10.1007/BF00003470.

URL https://link.springer.com/article/10.1007/BF00003470

[20] J. N. Phillips, J. Myers, Growth Rate of Chlorella in Flashing Light. 1, Plant Physiology 29 (2) (1954) $152-161$. URL https://www.ncbi.nlm.nih.gov/pmc/articles/PMC540481/

[21] S. Abu-Ghosh, D. Fixler, Z. Dubinsky, D. Iluz, Flashing light in microalgae biotechnology, Bioresource Technology 203 (Supplement C) (2016) 357-363. doi:10.1016/j.biortech.2015.12.057.

URL http://www.sciencedirect.com/science/article/pii/S0960852415016879

[22] S. Abu-Ghosh, D. Fixler, Z. Dubinsky, D. Iluz, Continuous background light significantly increases flashing-light enhancement of photosynthesis and growth of microalgae, Bioresource Technology 187 (Supplement C) (2015) 144-148. doi:10.1016/j.biortech.2015.03.119.

URL http://www.sciencedirect.com/science/article/pii/S0960852415004514

[23] H. Qiang, A. Richmond, Productivity and photosynthetic efficiency of Spirulina platensis as affected by light intensity, algal density and rate of mixing in a flat plate photobioreactor, Journal of Applied Phycology 8 (2) (1996) 139-145. doi:10.1007/BF02186317.

URL https://link.springer.com/article/10.1007/BF02186317

[24] P. J. Graham, B. Nguyen, T. Burdyny, D. Sinton, A penalty on photosynthetic growth in fluctuating light, Scientific Reports 7 (1) (2017) 12513. doi:10.1038/s41598-017-12923-1.

URL https://www.nature.com/articles/s41598-017-12923-1

[25] D. Mitra, J. H. van Leeuwen, B. Lamsal, Heterotrophic/mixotrophic cultivation of oleaginous Chlorella vulgaris on industrial co-products, Algal Research 1 (1) (2012) 40-48. doi:10.1016/j.algal.2012.03.002.

URL http://www.sciencedirect.com/science/article/pii/S2211926412000082

[26] K.-L. Yeh, J.-S. Chang, Effects of cultivation conditions and media composition on cell growth and lipid productivity of indigenous microalga Chlorella vulgaris ESP-31, Bioresource Technology 105 (Supplement C) (2012) 120-127. doi:10.1016/j.biortech.2011.11.103.

URL http://www.sciencedirect.com/science/article/pii/S0960852411017147

[27] X. Wu, J. C. Merchuk, A model integrating fluid dynamics in photosynthesis and photoinhibition processes, Chemical Engineering Science 56 (11) (2001) 3527-3538. doi:10.1016/S0009-2509(01)00048-3.

URL http://ww. sciencedirect.com/science/article/pii/S0009250901000483

[28] B. Cheirsilp, S. Torpee, Enhanced growth and lipid production of microalgae under mixotrophic culture condition: Effect of light intensity, glucose concentration and fed-batch cultivation, Bioresource Technology 110 (Supplement C) (2012) 510-516. doi:10.1016/j.biortech.2012.01.125.

URL http://www.sciencedirect.com/science/article/pii/S0960852412001496

[29] H. Ben Amor-Ben Ayed, B. Taidi, H. Ayadi, D. Pareau, M. Stambouli, Magnesium Uptake by the Green Microalga Chlorella vulgaris in Batch Cultures, Journal of Microbiology and Biotechnology 26 (3) (2016) 503-510. doi:10.4014/jmb.1507.07039.

[30] X. Chen, Q. Y. Goh, W. Tan, I. Hossain, W. N. Chen, R. Lau, Lumostatic strategy for microalgae cultivation utilizing image analysis and chlorophyll a content as design parameters, Bioresource Technology 102 (10) (2011) 6005-6012. doi:10.1016/j.biortech.2011.02.061.

URL http://www.sciencedirect.com/science/article/pii/S0960852411002677

[31] N. Hidasi, A. Belay, Diurnal variation of various culture and biochemical parameters of Arthrospira platensis in large-scale outdoor raceway ponds, Algal Research 29 (2018) 121-129. doi:10.1016/j.algal.2017.08.027.

URL https://www.sciencedirect.com/science/article/pii/S2211926417302217

[32] R. J. Porra, A simple method for extracting chlorophylls from the recalcitrant alga, Nannochloris atomus, without formation of spectroscopically-different magnesium-rhodochlorin derivatives, Biochimica et Biophysica Acta (BBA) Bioenergetics 1019 (2) (1990) 137-141. doi:10.1016/0005-2728(90)90135-Q.

URL http://www.sciencedirect.com/science/article/pii/000527289090135Q

[33] B. Demmig-Adams, W. W. Adams, The role of xanthophyll cycle carotenoids in the protection of photosynthesis, Trends in Plant Science 1 (1) (1996) 21-26. doi:10.1016/S1360-1385(96)80019-7.

URL http://www.sciencedirect.com/science/article/pii/S1360138596800197

[34] H. C. P. Matthijs, H. Balke, U. M. v. Hes, B. M. A. Kroon, L. R. Mur, R. A. Binot, , Biotechnology and Bioengineering 50 (1) (1996) 98-107. doi:10.1002/(SICI)1097-0290(19960405)50:1<98::AID-BIT11>3.0.CO;2-3.

URL https://onlinelibrary.wiley.com/doi/abs/10.1002/\%28SICI $\% 291097-0290 \% 2819960405 \% 2950 \% 3 \mathrm{~A} 1 \% 3 \mathrm{C} 98 \% 3 \mathrm{~A} \%$ 3AAID-BIT $11 \% 3 \mathrm{E} 3.0 . \mathrm{CO} \% 3 \mathrm{~B} 2-3$ 
[35] J. U. Grobbelaar, L. Nedbal, V. Tichý, Influence of high frequency light/dark fluctuations on photosynthetic characteristics of microalgae photoacclimated to different light intensities and implications for mass algal cultivation, Journal of Applied Phycology 8 (4) (1996) 335-343. doi:10.1007/BF02178576.

URL https://doi.org/10.1007/BF02178576

[36] H. K. Lichtenthaler, Vegetation Stress: an Introduction to the Stress Concept in Plants, Journal of Plant Physiology 148 (1) (1996) 4-14. doi:10.1016/S0176-1617(96)80287-2.

URL http://www.sciencedirect.com/science/article/pii/S0176161796802872

[37] P. G. Falkowski, J. LaRoche, Acclimation to Spectral Irradiance in Algae, Journal of Phycology 27 (1) (1991) 8-14. doi:10.1111/j.0022-3646.1991.00008.x.

URL https://onlinelibrary.wiley.com/doi/abs/10.1111/j.0022-3646.1991.00008.x 